\title{
Evaluation of Experts' Preferences for Selection of Suitable Industrialized Building Systems
}

\author{
Yidnekachew Daget ${ }^{1}$ and Hong Zhang $^{2}$ \\ ${ }^{1} P h D$ candidate, School of Architecture, Southeast University, China, email: tesmammay@yahoo.com \\ ${ }^{2}$ Professor, School of Architecture, Southeast University, China. email:zhangh555@aliyun.com
}

\begin{abstract}
Experts' preferences for building material selection are a largely under-explored part of a material research. This study evaluates the experts' preferences to identify the suitable industrialized building system for application in Addis Ababa. Ten industrialized building systems were considered as alternatives for comparison. These systems have been used in a housing development in different parts of the world. A relevant literature review and contextual analysis were conducted. An analytical hierarchy process and an Expert Choice Comparion platform were employed as a research technique and tool to evaluate the professionals' level of preferences with regard to the housing systems. The findings revealed the most important preferential attributes and the suitable industrialized building systems for an application in housing development. The decision criteria and the experts' preferential method used in this study can help decision-makers and housing developers in developing countries make effective evaluations and decisions.
\end{abstract}

\section{Introduction}

The material research regarding the adoption of industrialized building system (IBS) has increased driven by the project performance opportunities it offers particularly in terms of sustainability, 'lean' efficiency in design and rapid construction process [1,2]. Despite the significant potential, the adoption of IBS in the African context is slow. Improving the technology requires a better understanding of preferential factors influencing the selection of IBS in the context of unique constructionindustry characteristics [3]. Experts' preference study has the potential to enrich material evaluation research resulting in materials that meet the necessary attributes. However, it is largely under-explored parts of material research particularly its application for housing development. Consequently, research gaps have emerged relating to the systematic decision-making of IBS selection based on experts' preference. This paper contributes to this body of knowledge by conducting an experts' preference evaluation of proposed IBS for adoption to the city of Addis Ababa. As shown in Figure 1 , ten IBS were chosen for evaluation based on their application in a housing development.

The city of Addis Ababa has a high rate of population growth and urbanization resulted in massive pressure for rapid affordable housing development [4]. The housing market can not sufficiently provide the existing housing demand. One of the main challenges is the lack of systematic decision-making for the selection of suitable housing system [5]. This study can help to address the challenges in the given context.

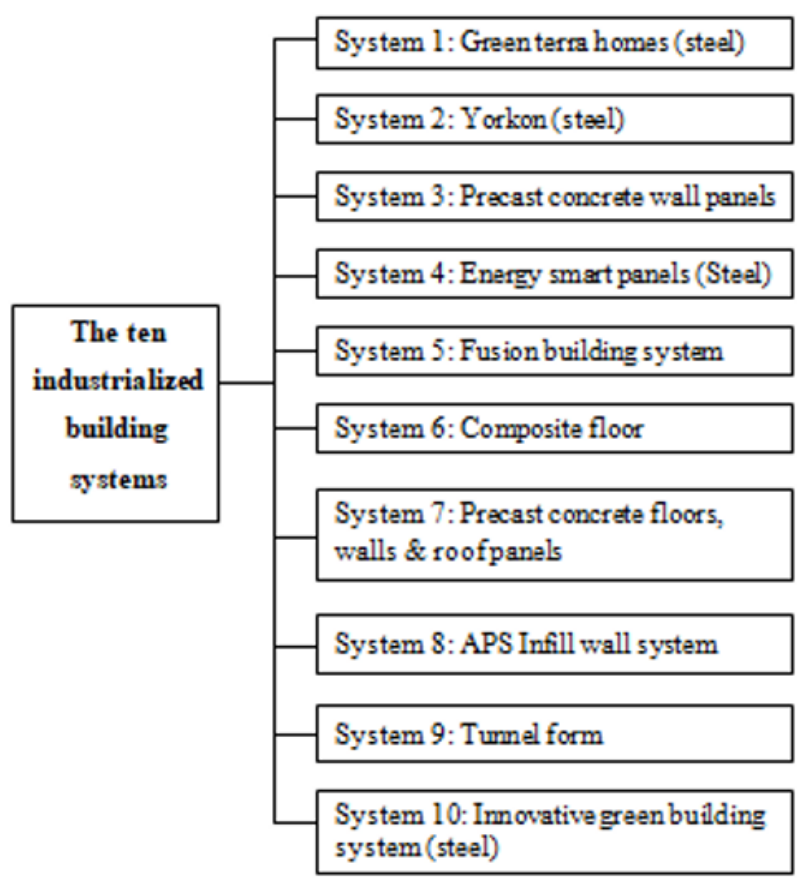

Figure 1. The ten industrialized housing systems.

\section{Decision-factors for building system selection}

Many studies have discussed that systematic decisionmaking is important for identifying the appropriate 
building system $[1,6]$. The decision-making process involves all stakeholders in the construction industry [7]. But, the processes of evaluating building systems are not well-studied regarding African context. Holistic decision criteria are important to consider in order to select the appropriate building system for application [8]. Kaitilla (1991) has shown that when choosing building materials, most people strive to fulfil tangible, intangible, and environmental variables [9]. A broad range of decision criteria other than the traditional technical and economic factors help to make effective decisions [10]. The environmental issue is one of the factors which broaden the scope of selection criteria [11]. It is becoming a growing concern in the construction industry.

Ilozor, Okoroh, Egbu, and Archicentre (2004) have presented that the success of a housing development is directly related to the right selection and adoption of suitable building systems [12]. This is well-known among off-site construction professionals where an incorrect decision resulted in failure due to the expensive cost of the imported material, lack of expertise and incompatibility to the local context [13]. Abdullah and Egbu (2010) have suggested that applications of new IBS are based on its recognized benefits in the construction industry [14]. Despite the pressing housing market's need in developing countries, companies that tried to introduce IBS met with failures. Therefore, it is significant to consider meaningfully all decision criteria in order to make right decision according to the local context.

\section{Methodology}

\subsection{Research design}

With the purpose of identifying the most appropriate among the ten building systems, the research employed survey-based study approach to comprehend a general opinion of practice on decision factors and to provide a thorough explanation $[15,16]$. Prior to the survey and the overall examination, an investigation was carried out with designated industrialized building experts from the local construction industry. The experts were interviewed to categorize the relevant factors, the encounters, and the resolutions for the implementation of IBS in Addis Ababa. Furthermore, they were invited to assess the importance, the reliability and the rationality of the questionnaire and made amendment prior to dissemination. Analytical hierarchy process (AHP) and Expert Choice Comparion (ECC) were used to rank the weighting of the factors and the alternatives to identify the suitable building systems. The outline of the research design is illustrated in Figure 2 .

\subsection{Decision factors and evaluation}

An overall survey was carried out to select professionals with prominent qualification to involve into the AHP examination. As the practice in the implementation of IBS is inadequate, there are limited numbers of experts with the essential proficiency in various groups in the Addis Ababa's building industry. Experts' examined the relevance of the proposed factors based on the holistic criteria listed by Pan, Wong, and Hui to make sure that comprehensive criteria were set for the building system assessment [2]. As listed in Table 1, the modified framework for various selection criteria was categorized under six level-one factors and thirty level-two factors to prioritize the weights of each factor.

This study employed the analytical hierarchy process (AHP) to assist the assessments of experts. AHP's consistency evaluation helps to provide trustworthy importance scales based on a relative rank of the selection criteria and the building systems [17]. The pairwise judgment of AHP assessment and examination was carried out using the platform of Expert Choice Comparion (ECC). ECC is an online-based decision support tool which delivers an easy access for decisionmakers to carry out the pairwise comparisons. Since the consistency ratio for individual expert judgment and the overall judgment was less than 0.1 , it is assumed that the evaluation was consistent and valid.

Table 1: The relevant decision factors

\begin{tabular}{lllllll}
\hline $\begin{array}{l}\text { Level } \\
\text { one }\end{array}$ & Building industry & Supply chain & Infrastructure & Sustainability & Users needs & Socio-cultural \\
\hline $\begin{array}{l}\text { Level } \\
\text { two }\end{array}$ & Labour & Material source & Transportation & environment & $\begin{array}{l}\text { Number of } \\
\text { stories } \\
\text { Flexibility }\end{array}$ & Public acceptance \\
& $\begin{array}{l}\text { Expertise and } \\
\text { experience } \\
\text { Construction } \\
\text { proficieny } \\
\text { Procurement }\end{array}$ & Machinery & Water & Pollution & Quality laws & Vested interests \\
& $\begin{array}{l}\text { Production } \\
\text { capacity } \\
\text { timnovation }\end{array}$ & Sewerage & Reusability & Affordability & Communication \\
& & waste disposal & efficiency & Site location & Culture \\
\hline
\end{tabular}




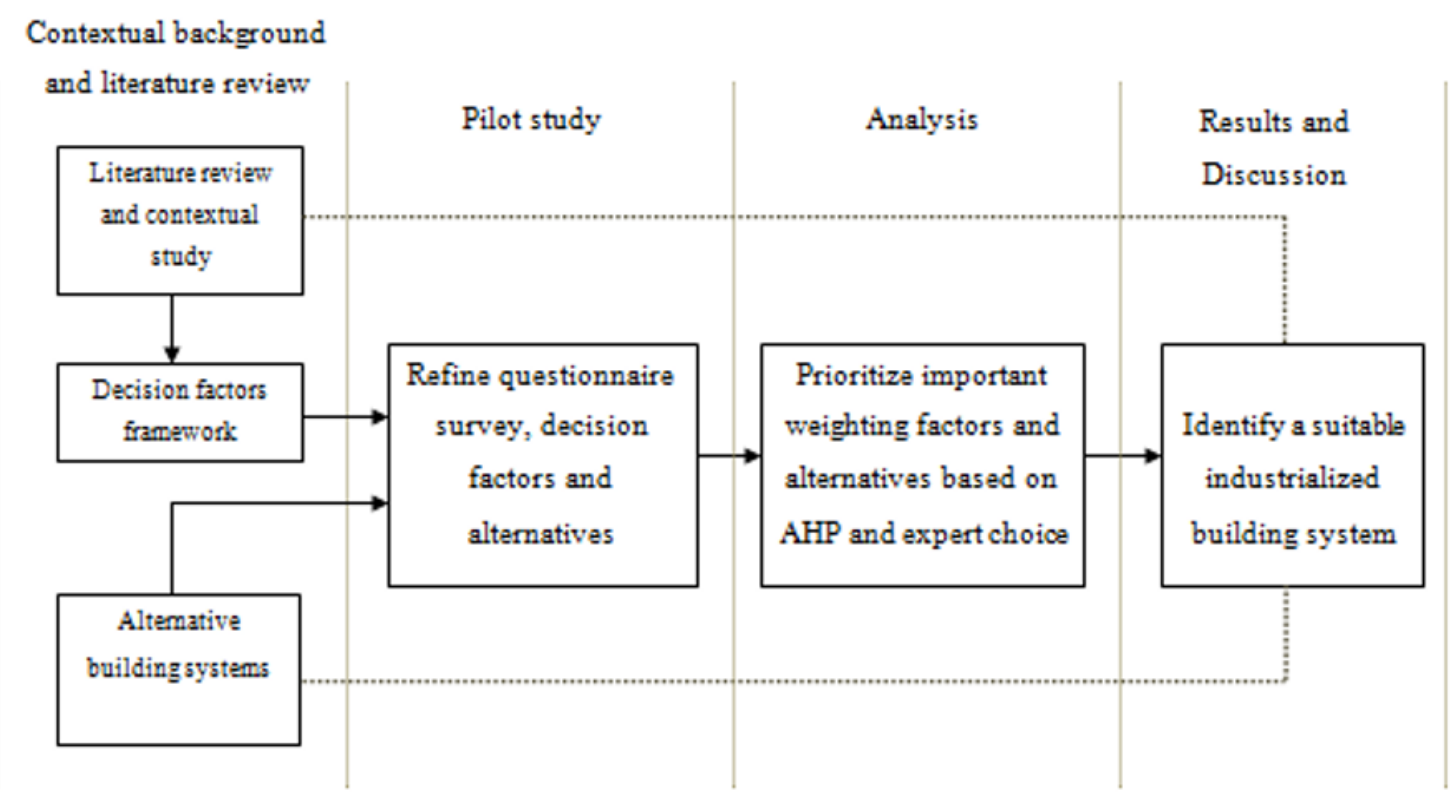

Figure 2. Research design diagram

\subsection{Data collection analysis}

Qualitative and quantitative data were collected. The qualitative data were gathered based on interviews and literature. The data were analyzed by the content analysis method, following structuring of the decision hierarchy. The quantitative data were collected and analyzed using Expert Choice Comparion (ECC). The general decisionmaking was examined through the following steps. As shown in Figure 3, primarily, the AHP decision model was structured into objectives, level-one factors, leveltwo factors, and alternatives to weigh the relevant factors and the alternatives. The pairwise comparison was carried out with ten selected experts using ECC to define priority vectors at level-one and level-two factors. Then, a numerical evaluation and normalization were done according to the assessment of the level of importance to identify the finalization of priorities. Lastly, a pairwise comparison was carried out by thirty identified experts and practitioners within the Addis Ababa construction industry who are key players in the precast manufacturers, suppliers, contractors, academician, engineers, and developers with an average of fifteen years of experience. The assessment indicated that the appropriateness of the ten housing systems was based on a pairwise judgment scale of 1-9 conducted to every level-two factors. Finally, the analyzed results of the alternatives were consequently weighed; then, the most appropriate IBS for Addis Ababa's housing development were selected.

\section{Results and discussion}

\subsection{Priority of decision factors}

As listed in Table 2, the finalized score for each level-one factor reveals that users needs, supply chain, and building industry are the most decisive factors for the selection of
IBS in Addis Ababa. These factors are the key component part of a housing construction industry. Previous studies also revealed that the top three factors have prime importance in many developing countries [2, 18]. These factors are related to the challenges of the provision of affordable housing. One of the main challenges is lack of appropriate materials with a required quality and affordable price. This is a common challenge in most developing countries as the ability of the population to pay for a decent housing is very low. This resulted in the situation where the majority of city dwellers live in poor housing conditions. Therefore, the application of cost-effective IBS not only contributes to the high rate of affordable housing development but also improves the housing conditions. In addition, the success of a large-scale housing development is also dependable on the availability of sufficient production capacity and supply of building components.

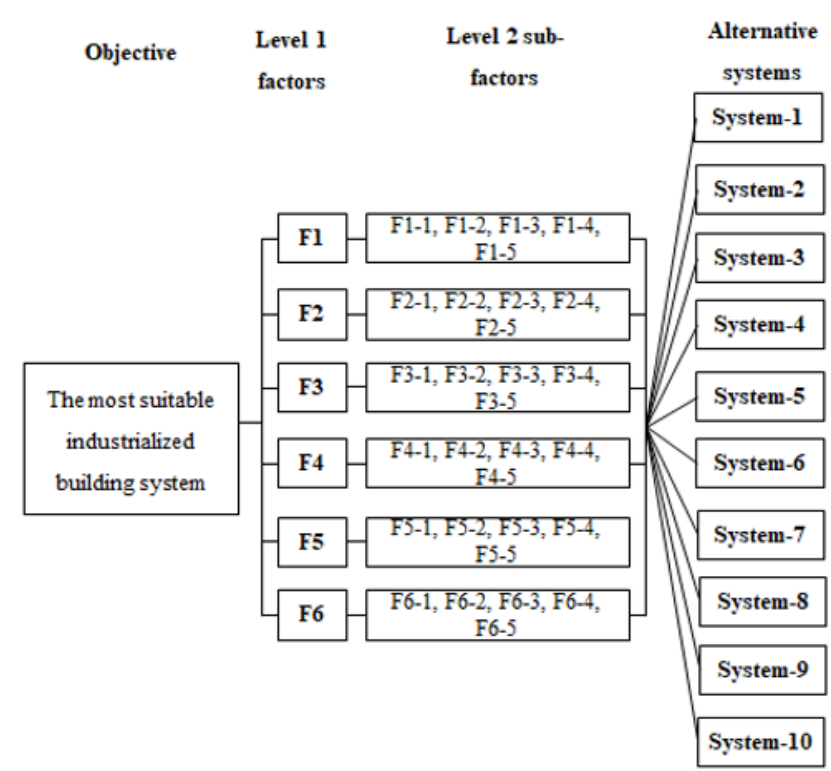

Figure 3. The Analytical Hierarchy Process (AHP structure). 
In Addis Ababa, the manufacturing capacity of developing new cost-effective materials and sufficient amount building components is not well developed [19]. Innovation is the best way to push forward the boundary of the performance of building components and the method of construction in order to solve the problem related to a wide application of IBS for affordable housing development. The problem is aggravated by the disorganized nature of the existing supply chain management. These challenges have become a bottleneck for an extensive long-term application of IBS [5]. However, the issue of sustainability was the least priority. This implies that the concern given for sustainability in housing development by construction professionals is less due to the pressing demand for housing with respect to the low capacity of the population to own a decent and quality housing.

The significance of the results of this study lies in experts' preference study. The level of importance of the decision-factors depended on the rich experience of the experts. As Wilson (1971) meant it is the reality of the view that people hold that ultimately determines choices. According to the central point of choice theory, any choice is intended to produce and advance pleasure, happiness, and benefit [20]. It is argued that choices produce satisfaction, especially when one chooses the item of highest utility value while discriminating against those with low utility value [21]. The priorities of the major decision criteria showed that the need and satisfaction of the customer got prime importance over the other. This is related to the utility value of the choice theory. In this sense, choices can be used to predict individual or group knowledge, attitudes, and values. In summary, the results showed that there is a wide range of factors influencing choices of building materials.

Table 2. Level-one and level-two factors analyzed ranking.

\begin{tabular}{lll}
\hline Level 1 & Priority Vector \% & Rank \\
\hline Building industry & 14.36 & 3 \\
Supply chain & 25.06 & 2 \\
Infrastructure & 9.60 & 5 \\
Sustainability & 4.59 & 6 \\
Users needs & 39.28 & 1 \\
Socio-cultural & 7.11 & 4 \\
\hline
\end{tabular}

\subsection{Priority of the ten IBS}

The priority rank of the ten IBS shows that the top three systems are the most suitable for Addis Ababa. As it is

Table 4: Characteristics of the suitable housing system

\begin{tabular}{|c|c|c|c|c|}
\hline Structural & Architectural & Industrialized process & Erection & Economic and social \\
\hline Durability & Flexibility in plan & Concrete components & Easy handling & Lower cost for high quality \\
\hline $\begin{array}{l}\text { Load bearing } \\
\text { components }\end{array}$ & Open systm & $\begin{array}{l}\text { Middle degree } \\
\text { mechanization }\end{array}$ & $\begin{array}{l}\text { Compatible with } \\
\text { conventional } \\
\text { system }\end{array}$ & $\begin{array}{l}\text { Adaptability to the needs of various } \\
\text { socio-economic groups }\end{array}$ \\
\hline $\begin{array}{l}\text { Fire, sound and } \\
\text { water proof }\end{array}$ & $\begin{array}{l}\text { Medium to high } \\
\text { rise residential }\end{array}$ & $\begin{array}{l}\text { Panelised, and frame and } \\
\text { infill techniques }\end{array}$ & $\begin{array}{l}\text { Rapid } \\
\text { construction }\end{array}$ & $\begin{array}{l}\text { Need for semi-skilled/ unskilled } \\
\text { labour }\end{array}$ \\
\hline
\end{tabular}

shown in Table 3, the three systems are all precast concrete systems. They are precast concrete slab and stairs (System 7), precast concrete column, beam, crosswalls, floors and stairs (System 6), and precast concrete wall panels (system 8). The findings from this exercise were very interesting indeed. As it is shown in Figure 4, after a close scrutiny of the materials that were chosen, two large, distinct groups emerged. On the one hand, those that scored highly on the preference list included all precast concrete systems. On the other hand, those that scored very low are all steel systems.

Table 3. Ranking of the alternative housing systems

\begin{tabular}{lll}
\hline $\begin{array}{l}\text { The ten industrialized housing } \\
\text { systems }\end{array}$ & $\begin{array}{l}\text { (Priority } \\
\text { Vector \%) }\end{array}$ & $\begin{array}{l}\text { Priority } \\
\text { ranking }\end{array}$ \\
\hline S1: Green terra homes (steel) & 5.96 & 10 \\
S2: Yorkon (steel) & 6.26 & 9 \\
S3: Precast concrete wall panels & 15.38 & 3 \\
S4: Energy smart panels (Steel) & 8.24 & 5 \\
$\begin{array}{l}\text { S5: Fusion building system (steel) } \\
\text { S6: Precast concrete column, }\end{array}$ & 7.79 & 6 \\
beam, cross-walls & 16.24 & 2 \\
$\begin{array}{l}\text { S7: Precast concrete slab and stairs } \\
\text { S8: APS Infill wall system }\end{array}$ & 17.22 & 1 \\
$\begin{array}{l}\text { S9: Tunnel form } \\
\text { S10: Innovative green building } \\
\text { system (steel) }\end{array}$ & 9.40 & 4 \\
& 6.62 & 8 \\
& & 7
\end{tabular}

As it is listed in Table 4, the common characteristics of the suitable IBS are load-bearing, easily erectable, sound and fireproof, good for application in medium to high rise residential buildings, and economical in large scale production. The systems are about $25 \%$ more productive than the conventional method of construction [22]. They are open systems that are compatible with both conventional and industrialized components. The three systems are semi-industrialized since they only provide parts of the building, and exclude the finishing and mechanical-electrical elements. One type of semiindustrialized process involves "pre-cutting" of housing components and partial assembly to reduce onsite labour and erection time resulting in panelized houses. Another type of semi-industrialization process involves 'frame and infill" techniques, which permit smaller prefabricated panels to be installed in a structural frame [23]. The top ranking systems use both the panelized, and frame and infill technique and perceived as the favoured housing system in Addis Ababa. 

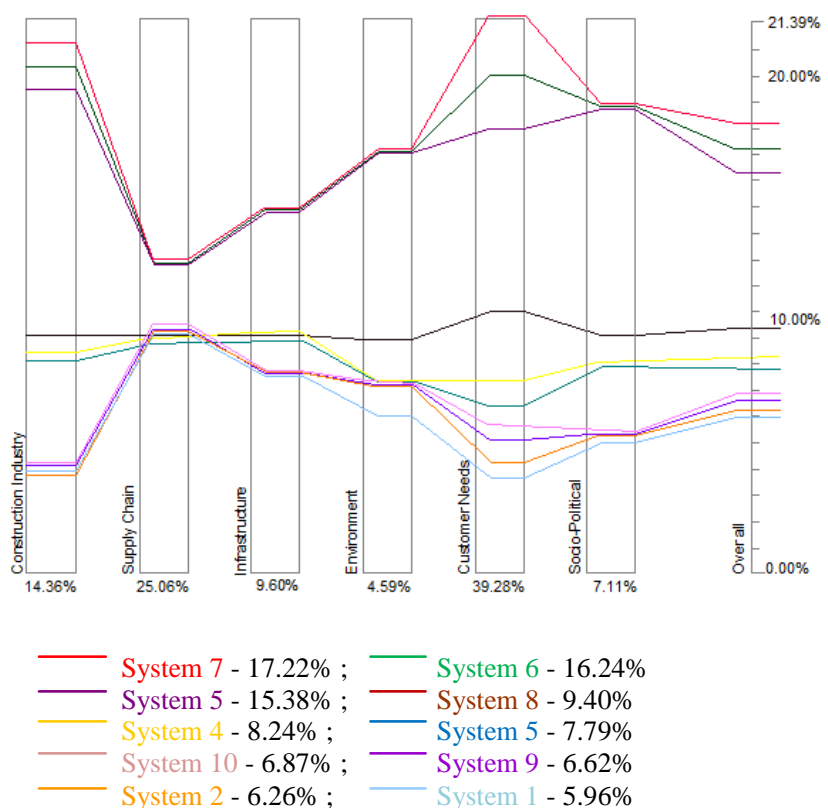

Figure 4: Perfomance analysis of the ten IBS with respect to the level-one decision-factors

As it is shown in Figure $5 a, 5 b$, and $5 c$, the top three systems has priority percentage in terms of the three most important decision factors. These factors are some of the main reasons for introducing IBS in housing development as well as to solve the housing supply shortage by improving construction productivity. These imply that the three systems positively contribute to improving the productivity rate of affordable housing construction as well as enhancing the capacity of the local construction industry for high-quality products and workmanship. The products are manufactured based on the overall plans, systematic designs, uniform standards, mass production and standardized shapes. The modular component system can be adjusted to varying financial capabilities of beneficiaries. Local input materials can be used for production with a request for semi-skill specialization. The components can also be better utilized and wastage kept to a minimum. In addition, it significantly reduces the use of formwork compare to the conventional systems. In Addis Ababa, wooden formwork is commonly used for construction and has contributed to significant loss of the natural forest [24]. Therefore, housing systems which reduce the use of formwork are appropriate for Addis Ababa. This made the housing systems viable option to apply them for cost-effective mass housing development.

However, these systems have limitations, such as lack of sufficient supply chain, jointing problems; heavyweight, capital-intensive production process, costly transportation and lack of flexibility to use. Continuous improvement is required to prevent cracking around the joints. In general, the prevailing incomplete supply chain of building materials and components in the local construction industry and the poor manufacture capacity is another challenge for the wide application of the housing systems.

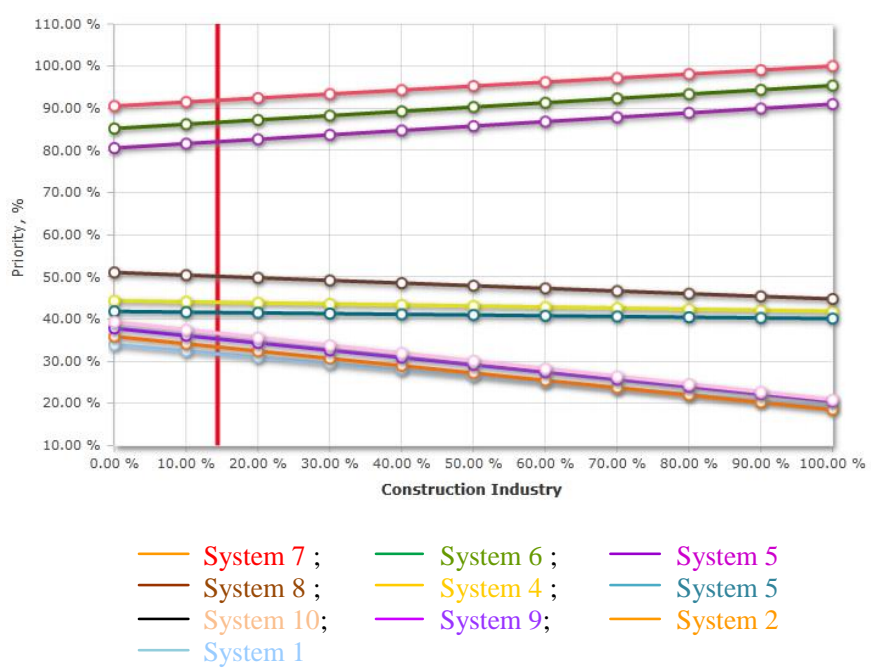

Figure 5a: IBS performance based on the construction industry

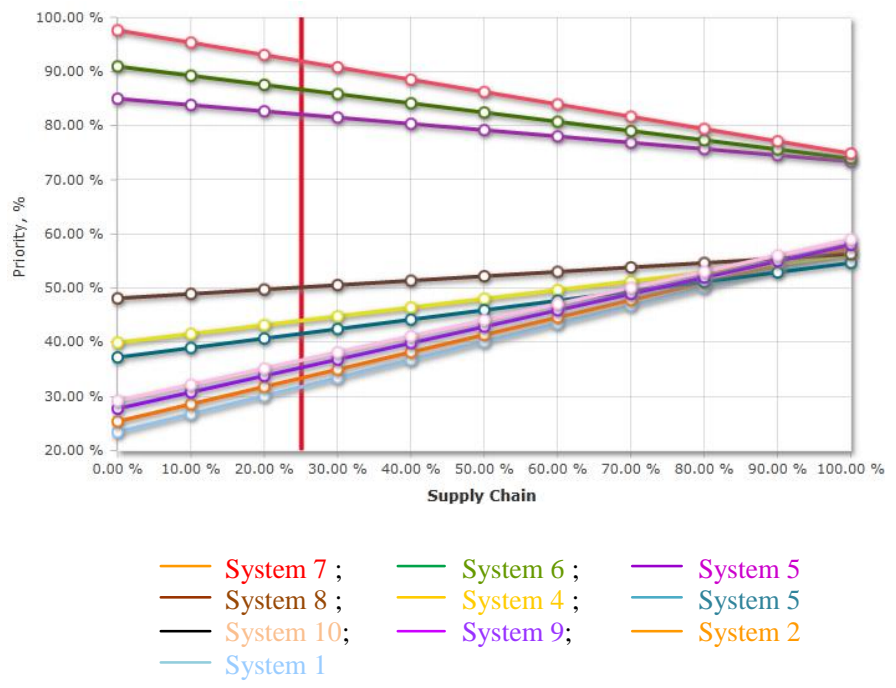

Figure 5b. IBS performance based on the Supply chain.

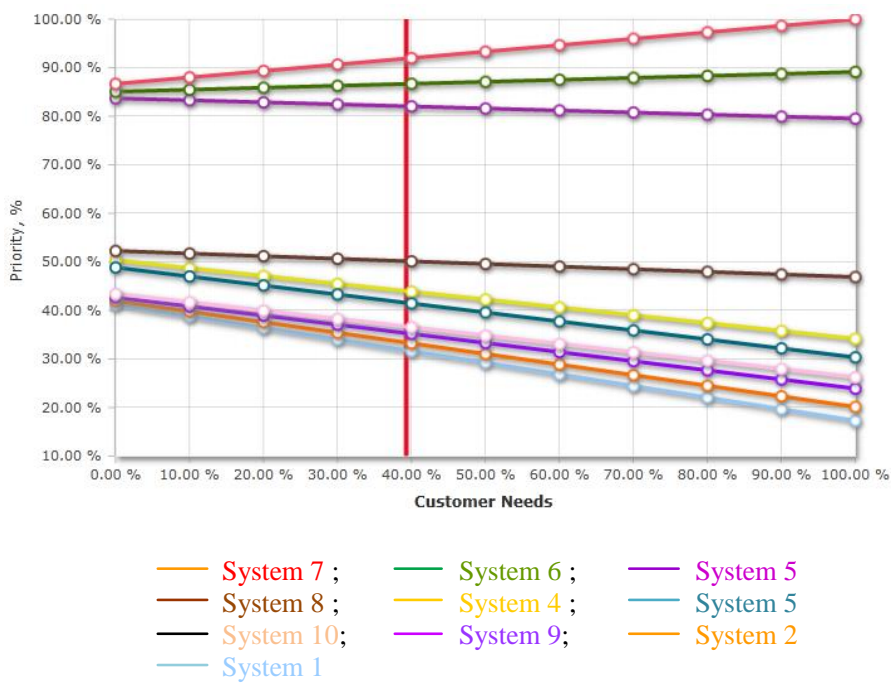

Figure 5c. IBS performance based on the customer needs. 


\section{Conclusions}

Material research has suffered from an over-emphasis on the engineering performance of new materials and the concomitant under-emphasis of experts' perceptual and holistic decision-criteria. In this study, experts' preferences for materials were explored. The study demonstrates the utility of research on the influential attributes of material and the systematic decision-making for the adoption of suitable IBS. The findings revealed that three types of precast concrete systems are suitable for application in Addis Ababa. These IBS systems are selected due to their characteristics of being economically competitive, use of the local material resource, less use of energy, and ease of constructability, and compatibility with conventional components. The AHP rankings of selected IBS are in line with the current construction status in Addis Ababa. However, they have limitations such lack of sufficient supply chain, and jointing problems.

The Addis Ababa market is still underdeveloped, owing to lack of advanced technologies and enough investment capital. To succeed dealing with these challenges, the Addis Ababa city government has to take actions in order to develop the capacity of quality products and to support developers to improve these precast concrete systems according to the local context. Hence, deciding the most suitable IBS for Addis Ababa needs to take into account the affordability, social benefits and environmental aspects of the local context. In addition, the important decision factors should be considered as part of holistic criteria for better design and organization to apply IBS. The Experts' preferences research method used can help to contribute to the body of knowledge of material science and used as a tool for housing developers in order to make effective decisions.

\section{References}

1. A.S. Zakaria, T. Gajendran, T. Rose, G. Brewer, AEDM. 1-24 (2017)

2. Y. Pan, F.K.W. Wong, E.C.M. Hui, Modeling risk management in sustainable construction. 161-8 (2011)
3. F.W. Kabo, Low cost housing design and provision : a case study in Kenya. University of Michigan. (2006)

4. UN-Habitat, The state of Addis Ababa. (2017)

5. H. Teka, National report on housing and sustainable urban development. (2014)

6. A.G.F. Gibb, Off-site Fabrication. Whittles Publishing. (1999)

7. W. Pan, A.G.F. Gibb, A.R.J. Danity. INT J HOUS SCI APPL. 32 (1), 61-79 (2008)

8. S. Conards, A. Othman, Industrialized building systems for low-cost housing projects. Lamber (2011)

9. S. Kaitilla, ARCHIT BEHAV. 7(3), 205-22. (1991)

10. K. Nassar, W. Thabet, Y. Beliveau, AUTOMAT CONSTR. 12 (5), 543-60 (2003)

11. W. Pan, A.G.F. Gibb, A.R.J. Dainty, CONSTR MANAGE ECON. 25(2), 183-94 (2007)

12. B.D. Ilozor, M.I. Okoroh, C.E Egbu, BUILD ENVIRON PLANN. 39(3), 327-37 (2004)

13. D. Richards, Which offsite system is right for you. (2004)

14. M.R. Abdullah, C.O. Egbu, CIB World conference. (2010)

15. A. Bryman. Research methods and organization studies. Unwin hyman. (1989)

16. S.L. Jackson. Research Methods and statistics. Cengage learning. (2015)

17. T.L. Saaty, INT J SERV SCI. 1(1). (2008).

18. C. Atkinson, A. Yates, M. Wyatt, Sustainability in the built environment. Watford. (2009)

19. HDAP, Ten years plan for housing development and administration. (to be published)

20. M. Wilson, Religions and the transformation of society. Cambridge university press. (1971)

21. C. Norberg-Schulz, Intentions in architecture. Allen Unwin ltd. (1963)

22. Y.H. Pan, Application of industrialized housing system in major cities in China. Hongkong polytehnique university. (2007)

23. I. Majzub. System building hardware. Wiley. (1980)

24. S. Kebede, The impact of alternative construction tehnology on condominium housing project. Ethiopian civil service university. (2013) 